\title{
Verification of CyberKnife Dose Distributions with Gafchromic EBT2 Film
}

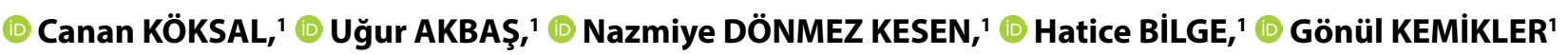

'Department of Medical Physics, İstanbul University Institute of Oncology, İstanbul-Turkey

\begin{abstract}
OBJECTIVE
CyberKnife (CK) system is more sophisticated than the conventional methods; therefore, strict quality assurance tests are required. To ensure that the treatment is delivered as planned, the verification of the treatment plan should be performed with specific dosimetry tools. This study aims to verify the CK treatment plans generated for patients with intracranial tumors using Gafchromic EBT2 film.
\end{abstract}

\section{METHODS}

The CK plans of ten patients with intracranial tumors were selected for this study. The plans were transferred to computed tomography images of the anthropomorphic head phantom. The phantom containing the film was irradiated using $6 \mathrm{D}$ skull tracking method. The dose distributions calculated by TPS were compared with those measured by film using gamma analysis method.

\section{RESULTS}

In the gamma-index method, the average passing rate for the $3 \% / 3 \mathrm{~mm}$ criterion was found to be $93.9 \pm 4.8 \%$. The gamma passing rate for Iris-collimator-based plans yielded slightly lower than that for fixed-collimator-based plans.

\section{CONCLUSION}

In conclusion, this study indicates that Gafchromic EBT2 film is a utilizable dosimeter for quality assurance of the CK treatment plans. It is recommended to use the EBT2 film for verification of the CK treatment plans before delivery to the patient.

Keywords: CyberKnife; film dosimetry; gamma evaluation; quality assurance.

Copyright $\odot$ 2018, Turkish Society for Radiation Oncology

\section{Introduction}

Stereotactic radiosurgery (SRS), one of the advanced radiotherapy techniques, is capable of delivering extremely high radiation dose to the target in a treatment fraction. It was initially implemented in 1967 in Sweden for the treatment of intracranial targets with GammaKnife.[1] Today, thanks to the rapid development in technology, gantry-based and robotic-based linear accelerators are used for the SRS treatments of both intracranial and extracranial tumors. Unlike conventional radiotherapy techniques, the lesions treated with the SRS technique are very small in size. Mostly, they are in proximity to vital organs. In SRS, very small fields or beamlets are used to achieve dose distributions conforming more tightly to the precisely defined tumor shape by reducing the doses to the critical organs.[2]

The CyberKnife (CK) is a unique system comprising of a robotic-based linear accelerator. In the CK treatments, for the rapid fall-off of dose between the target and normal tissues, 150-300 non-isocentric beams from different node positions are delivered to 
the target with a compact linear accelerator mounted on a robotic arm. Throughout the treatment delivery, tumor position is monitored through an imaging system. The CK treatment delivery is very complicated due to all these factors, and hence, strict quality assurance (QA) tests are required.[3]

The periodic machine QA guidance has been explained in the report from AAPM Task Group 135. In this report, it is recommended that the automated-quality-assurance (AQA) test and the end-to-end (E2E) test be routinely carried out for mechanical accuracy. The implementation processes of both tests are described in detail. The AQA test is used for determining isocentric targeting accuracy. The E2E test is used to check the overall targeting accuracy of the CK system. They cannot be used for determination of dose accuracy for treatment plans of non-isocentric patient. It is also recommended that the delivery quality of assurance (DQA) test, which assesses both spatial and dosimetric accuracy of delivery, is performed as a part of the machine commissioning and monthly QA using film or detectors. [4] Additionally, because of the complexity of treatment delivery, verification of the planned dose distribution before delivering it to the patient has become necessary in the CK treatments. Another crucial point that requires implementing pretreatment QA is that the non-isocentric beam directions are unique for each patient. Nevertheless, there are no published procedures about the beam-by-beam QA for the CK treatments.

The radiochromic film is an ideal dosimeter to obtain dose distributions in high dose gradient regions due to its high spatial resolution and low spectral sensitivity. Films provide two-dimensional (2D) dose maps of treatment plans. Regarding spatial resolution, they are superior to other $2 \mathrm{D}$ radiation detectors. [5] The practice of film dosimetry on verification of intensitymodulated radiotherapy (IMRT) plans is well reported. However, verification of the CK dose distributions using film dosimetry is not commonly performed in the clinical practice for routine implementations due to insufficient detailed procedures and investigations.

The purpose of this study was to verify the CK dose distributions generated for patients with intracranial tumors using Gafchromic EBT2 film.

\section{Materials and Methods}

All measurements were performed on the CK radiosurgery system. The system comprised of a linear accelerator, robotic manipulator, and X-ray imaging sys- tem. The linac is mounted on a multi-jointed robotic manipulator that has six degrees of freedom of movement. This freedom of movement allows each beam to be directed to different points. The X-band linear accelerator produces an X-ray beam of $6 \mathrm{MV}$ with a dose rate of $800 \mathrm{MU} / \mathrm{min}$. Twelve fixed circular collimators ranging from $5 \mathrm{~mm}$ to $60 \mathrm{~mm}$ in diameter at $800 \mathrm{~mm}$ SAD are used for secondary collimation. These collimators can be changed either manually or automatically. Alternatively, Iris variable aperture collimator is available that can provide the same 12 field sizes without the need for switching secondary collimators during the treatment. The imaging system consists of a pair of ceiling-mounted kilovolt X-ray tubes and a pair of floor-mounted amorphous silicon X-ray detectors. This system together with tracking methods enables us to monitor tumor or patient movement. Any changes in the tumor or patient position are automatically adjusted during the treatment delivery. The appropriate tracking method for the patient is selected according to bony anatomy, fiducial markers, or soft tissue contrast, while the treatment plan is generated by MultiPlan treatment planning system (TPS).[3] In this study, mechanical accuracy tests of the machine (AQA and E2E) were performed prior to the verification of intracranial CK dose distributions.

\subsection{Film Calibration}

In this study, GafchromicEBT2 (Lot no.: A04141003BB) (ISP, International Specialty Products, ABD) film was used. The EBT2 films comprise a single active layer with a nominal thickness of $30 \mu \mathrm{m}$. The active part of the film is asymmetrically located between the layers. The substrate is made of polyester with thickness of 175 $\mu \mathrm{m}$. There are three protective sheets with a total thickness of $80 \mu \mathrm{m}$ over the active layer. This non-symmetrical layer configuration causes different film responses for the same scanning orientation when different sides of the films are facing the scanner. The measurable dose range of the EBT2 film was 1-800 cGy.[6] Gafchromic EBT2 film is a suitable dosimetry tool for use in intensity-modulated radiotherapy (IMRT) treatment verification because of their nearly constant dose rate and energy independence.[7]

First, a calibration curve was created before using the films to get a dose map related to the plans. Gafchromic films were cut into $2 \times 2 \mathrm{~cm}^{2}$ pieces, and they were placed between the solid water slab phantoms at a depth of $5 \mathrm{~cm}$. The source to film distance was $100 \mathrm{~cm}$. Films were oriented perpendicular to the central axis of the beam, and they were irradiated with doses rang- 
ing from 1 to $800 \mathrm{cGy}$ at a $10 \times 10 \mathrm{~cm}^{2}$ field size using a photon energy of $6 \mathrm{MV}$. An unexposed film was used to assess the background. All films were scanned using a flatbed scanner (Epson 10000XL America Inc., Long Beach, CA) on the following day to provide maximum post-irradiation darkening.[8] Films were put on the scanner bed in the same orientation. Then, ImageJ software was used to separate the films into the blue, green, and red color channels; the red color channel was chosen due to its high contrast. The optical densities (ODs) of exposed films were acquired using the MEPHYSTO $\mathrm{mc}^{2}$ software program (PTW-New York Corp., Hicksville, NY). The net OD was obtained by subtracting OD for the background film from measured OD. The calibration curve was generated by plotting the net ODs of irradiation films against the known radiation doses.

\subsection{D Dose Distribution Measurements}

The anthropomorphic head phantom was used for film dosimetry. The phantom mimics the bone structures and soft tissues of an adult human head. A phantom with six RW3 mini plates (sized $6 \times 9 \mathrm{~cm}^{2}$ ) each of which consists of $1 \mathrm{~cm}$ thickness was prepared. Gafchromic EBT2 film was put in the center of this phantom. Then, the prepared mini phantom was placed in the special cavity of the anthropomorphic head phantom (Fig. 1). The phantom's computed tomography (CT) images were acquired in 1-mm-slice thickness and transferred to the MultiPlan v. 4.6 (Accuray, Sunnyvale, CA, USA) TPS. The phantom was immobilized with a blue patient support cushion during both CT imaging and irradia- tion. The CK plans of ten patients with intracranial tumors created using the ray-tracing algorithm through the sequential optimization process were selected for this study. Five plans from the selected ten patient treatment plans were generated using Iris collimator, and the other five plans were created using fixed collimator. Data related to plans are described in Table 1. Ten plans were transferred to the CT images of the anthropomorphic head phantom. The isodose curves were centered on the mini phantom. Then, dose distributions on phantom were recalculated with high resolution, and they were then saved (Fig. 2). Finally, the head phantom containing the film was irradiated using $6 \mathrm{D}$ skull tracking system that used skeletal anatomy to position the radiation beam. Films were scanned after $24 \mathrm{~h}$, and 2D dose maps were obtained using the calibration curve in the PTW Verisoft program. The dose distributions in the axial plane calculated by TPS were compared with those measured by film using gamma analysis, which is a quantitative benchmark based on dose difference (DD) and distance-to-agreement (DTA) criteria.[9] The $2 \% / 2 \mathrm{~mm}, 3 \% / 3 \mathrm{~mm}, 4 \% / 4 \mathrm{~mm}$, and $5 \% / 5 \mathrm{~mm}$ gamma criteria were evaluated, and the $10 \%$ lower dose threshold was implemented.

\section{Results}

2D dose distributions of the ten intracranial plans were obtained by using Gafchromic EBT2 film. Dose maps measured by films were compared with those calculated by TPS using the gamma-index method in the PTW Verisoft software program (Fig. 3). Four
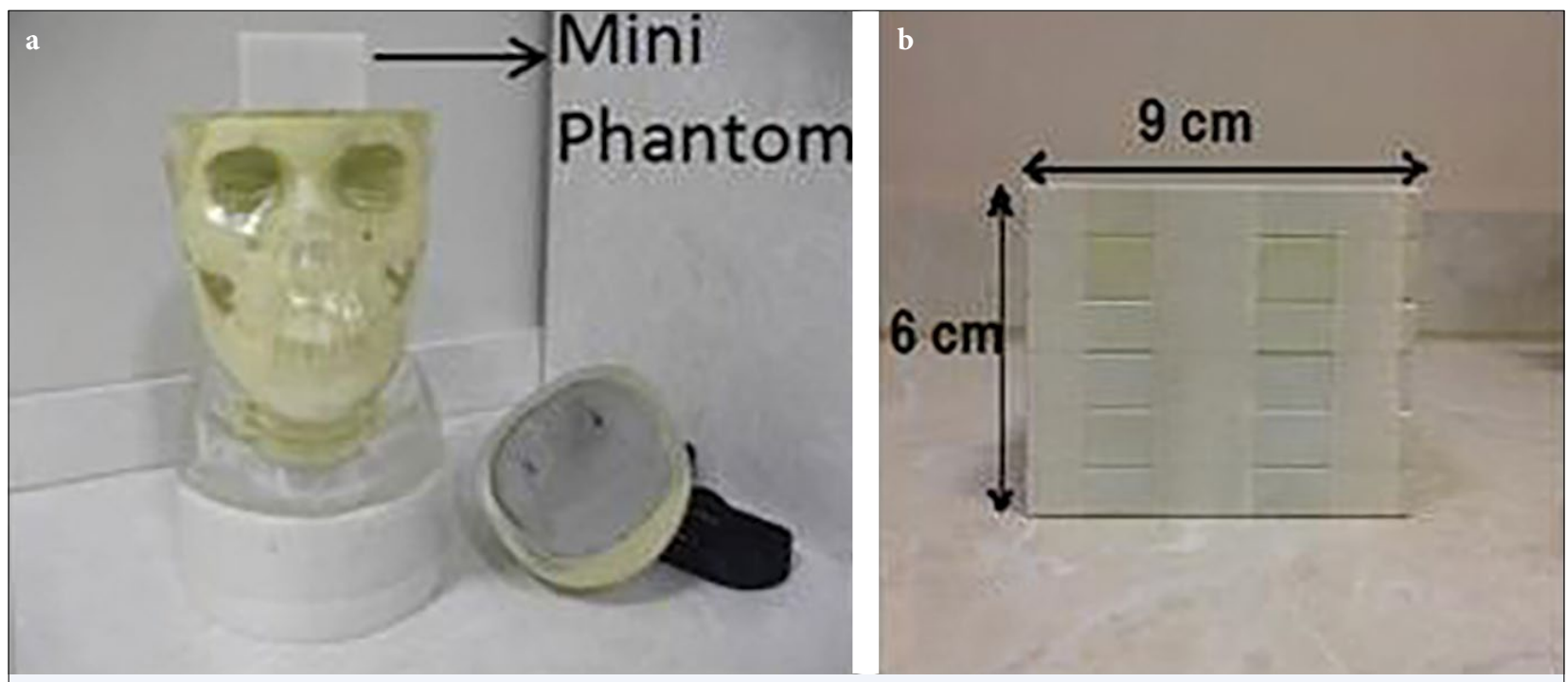

Fig. 1. (a) The anthropomorphic head phantom, (b) mini RW3 phantom. 
Table 1 Data related to plans

\begin{tabular}{lcccc} 
Intracranial Plan & Size of Collimator $(\mathbf{m m})$ & Collimator Type & Number of Beams & Number of Nodes \\
\hline Patient 1 & 10 & FIXED & 110 & 98 \\
Patient 2 & $10,12.5$ & FIXED & 150 & 88 \\
Patient 3 & 12.5 & FIXED & 103 & 86 \\
Patient 4 & 10,15 & FIXED & 85 & 75 \\
Patient 5 & 10,20 & FIXED & 96 & 90 \\
Patient 6 & 10 & IRIS & 116 & 102 \\
Patient 7 & $7.5,10,12.5$ & IRIS & 95 & 92 \\
Patient 8 & $10,12.5,15$ & IRIS & 106 & 74 \\
Patient 9 & $10,12.5,15,20$ & IRIS & 133 & 72 \\
Patient 10 & $12.5,15,20$ & & & 103 \\
\hline
\end{tabular}

Table 2 Gamma analysis results for ten intracranial CK treatment plans

DD/DTA

\begin{tabular}{|c|c|c|c|c|}
\hline \multirow[b]{2}{*}{ Plans } & \\
\hline & $2 \% / 2 \mathrm{~mm}$ & $3 \% / 3 \mathrm{~mm}$ & $4 \% / 4 \mathrm{~mm}$ & $5 \% / 5 \mathrm{~mm}$ \\
\hline Patient 1 & 77.1 & 95.4 & 99.4 & 99.9 \\
\hline Patient 2 & 83.8 & 97.7 & 99.8 & 100 \\
\hline Patient 3 & 79.5 & 97.9 & 99.9 & 100 \\
\hline Patient 4 & 70.9 & 92.6 & 97.3 & 99.2 \\
\hline Patient 5 & 86.1 & 95.9 & 99.7 & 100 \\
\hline Patient 6 & 81.9 & 96.8 & 99.3 & 100 \\
\hline Patient 7 & 74.4 & 92.6 & 98.2 & 99.8 \\
\hline Patient 8 & 76.6 & 95.8 & 99.5 & 100 \\
\hline Patient 9 & 55.7 & 80.4 & 91.2 & 95.8 \\
\hline Patient 10 & 76.0 & 93.7 & 98.2 & 99.6 \\
\hline Mean \pm SD & $76.2 \pm 8.5$ & $93.9 \pm 4.8$ & $98.3 \pm 2.6$ & $99.4 \pm 1.3$ \\
\hline
\end{tabular}

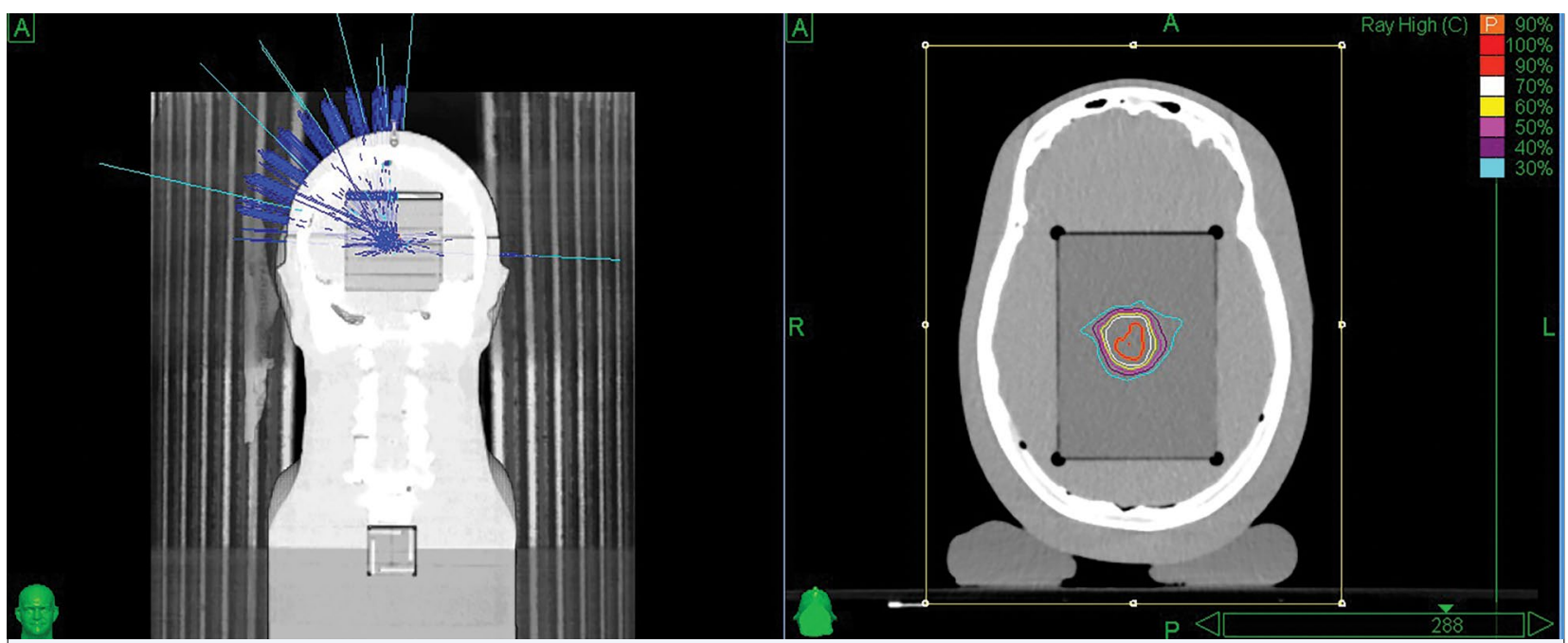

Fig. 2. Transfer of patient plan to the anthropomorphic head phantom.

gamma criteria were analyzed for $50 \%$ and above dose distributions, that is, $2 \% / 2 \mathrm{~mm}, 3 \% / 3 \mathrm{~mm}, 4 \% / 4 \mathrm{~mm}$, and $5 \% / 5$. The gamma passing rates for each intracra- nial plan are shown in Table 2 . The mean \pm standard deviation (SD) passing rate for the $2 \% / 2 \mathrm{~mm}, 3 \% / 3$ $\mathrm{mm}, 4 \% / 4 \mathrm{~mm}$, and $5 \% / 5 \mathrm{~mm}$ criteria are found to be 


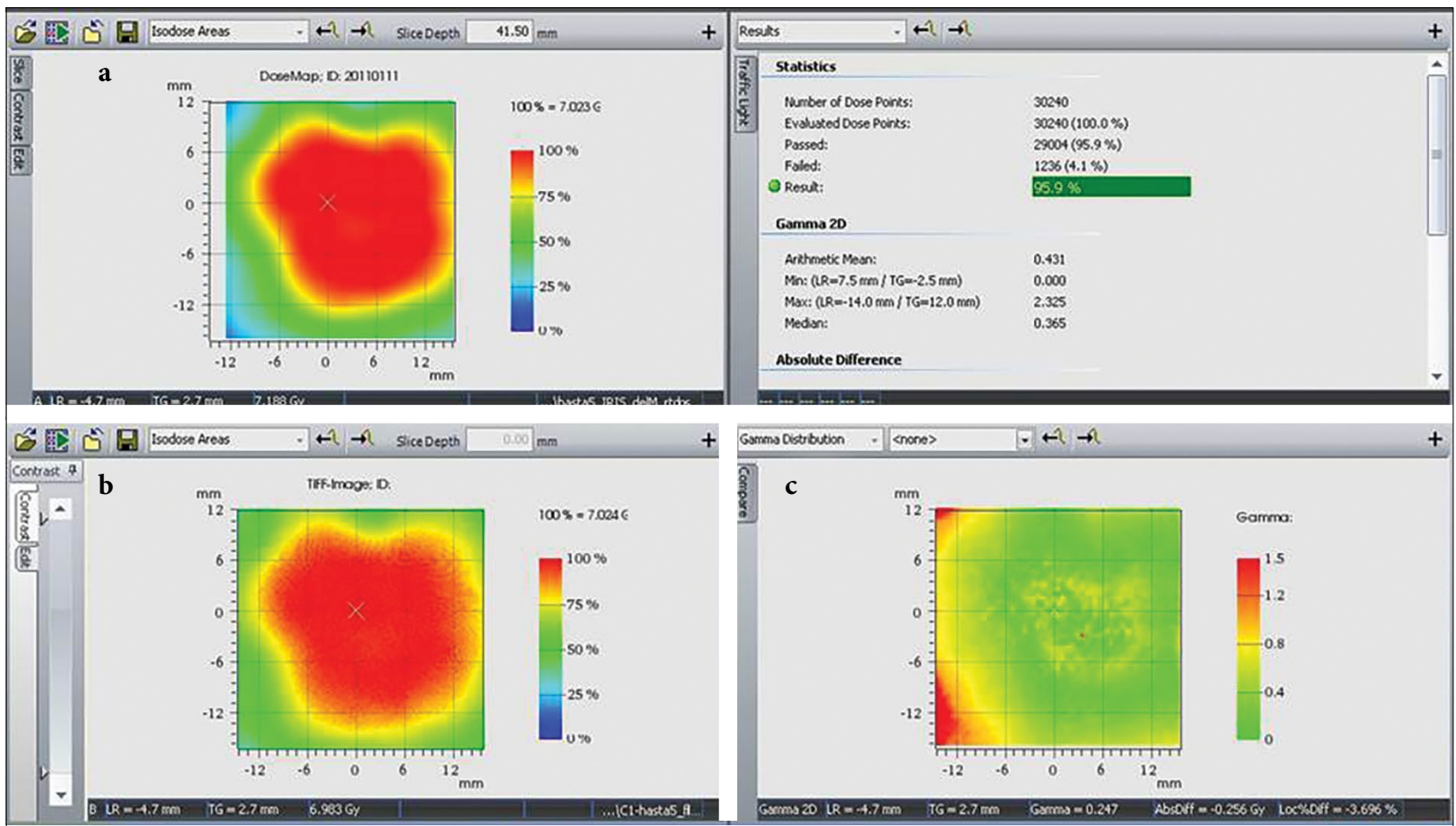

Fig. 3. Comparison of 2D dose distribution for patient 5: (a) calculated dose distribution (cGy); (b) measured dose distribution (cGy); (c) gamma distribution in gamma evaluation method.

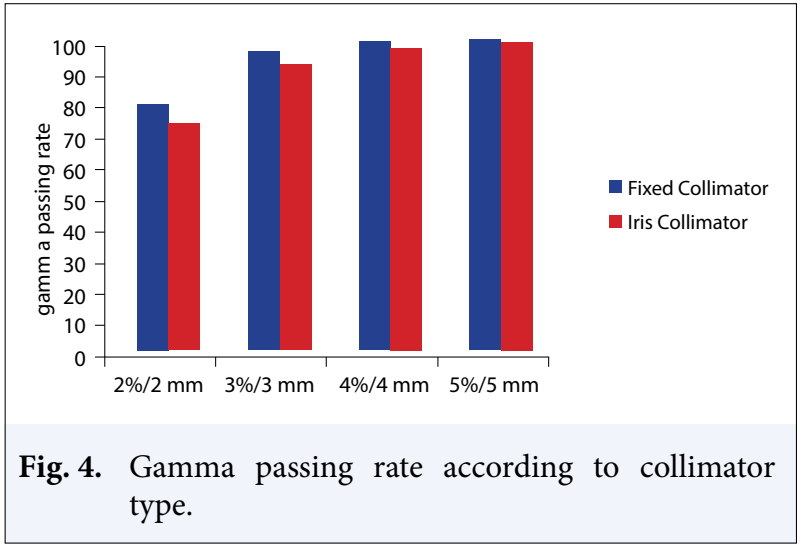

$76.2 \pm 8.5 \%, 93.9 \pm 4.8 \%, 98.3 \pm 2.6 \%$, and $99.4 \pm 1.3 \%$, respectively.

For five plans based on fixed collimator, the calculated gamma index with pass criteria $2 \% / 2 \mathrm{~mm}$, $3 \% / 3 \mathrm{~mm}, 4 \% / 4 \mathrm{~mm}$, and $5 \% / 5 \mathrm{~mm}$ are $79.5 \pm 5.6 \%$, $95.9 \pm 2.1 \%, 99.2 \pm 1.1 \%$, and $99.8 \pm 0.4 \%$, respectively. For other five plans based on Iris collimator, the gamma pass rates using same criteria are $72.9 \pm 10.0 \%$, $91.9 \pm 6.6 \%, 97.3 \pm 3.5 \%$, and $99.0 \pm 1.8 \%$, respectively. The percentage gamma-index value for Iris-collimatorbased plans yielded slightly lower than that for fixedcollimator-based plans (Fig. 4).

\section{Discussion}

The CK system, an image-guided robotic radiosurgery system, is used for the treatment of both intracranial and extracranial lesions. Unlike conformal radiotherapy, a very high radiation dose is delivered to the tumor in a single or few fractions with noncoplanar narrow beams in the CK treatment. One of the most important benefits of the CK treatment is that the dose outside the target volume falls off rapidly, and thus minimizing the doses to critical organs is achieved. Even the slightest failure in the treatment planning, delivery, or dosimetry can irretrievably affect the treatment outcomes. Therefore, to ensure that the treatment is delivered as planned, the verification of treatment plan should be performed with specific dosimetry tools. There are limited studies concerning performing QA of the treatment plan using film dosimetry.

Gafchromic films have been frequently used for patient-specific QA. Kairn et al. reported that Gafchromic EBT2 could be used for QA of complex IMRT treatment because of its high spatial resolution and minimal angular dependence of response. [7] Chan et al.[10] showed that Gafchromic EBT2 film is a useful dosimeter in patient-specific QA for 
linear accelerator-based SRS plans. In this study, 2D dose distribution verification of ten intracranial CK plans was performed using Gafchromic EBT2 film. The gamma analysis tool, which uses the dose and distance criteria simultaneously, provides a numerical gamma index as a result of the comparison of $2 \mathrm{D}$ dose measurements with the TPS calculations. There is no standard gamma-index criterion for plan verification. In general, the $3 \% / 3 \mathrm{~mm}$ criterion is used for IMRT patient-specific QA.[11,12] In our study, the gamma passing rate for the $2 \% / 2 \mathrm{~mm}, 3 \% / 3 \mathrm{~mm}$, $4 \% / 4 \mathrm{~mm}$, and $5 \% / 5 \mathrm{~mm}$ criteria was $76.2 \%, 93.9 \%$, $98.3 \%$, and $99.4 \%$, respectively. In our study, the gamma passing rate was lowest for patient 9. It was observed that the failed points were at the field edge and out of the $50 \%$ isodose distribution. Mardirossian et al.[13] evaluated dose distributions of eight patient plans generated in MultiPlan TPS using film dosimetry; the gamma-index value of $3 \% / 2 \mathrm{~mm}$ criterion was found to be $98.6 \%$ in their study. Bellec et al.[14] performed plan delivery quality assurance (DQA) test for the CK plans using Gafchromic EBT3 films. In their research, pre-treatment plans for 350 intracranial and extracranial cases were created on BabyBlue phantom. The gamma pass rate with criteria of $3 \% / 1.5$ $\mathrm{mm}$ was found to be $85 \%$. However, the decision as to which gamma-index criterion should be applied for QA of the CK treatment plan is a challenging issue. The gamma passing rate for $2 \% / 2 \mathrm{~mm}$ criterion was worst in our study. Minor setup errors and uncertainties in film analysis may have adversely affected the results because there were high dose gradient regions in the CK plans. Furthermore, non-isocentric beams from different angles may have caused uncertainties in the response of film. On the other hand, there is a good agreement for the $3 \% / 3 \mathrm{~mm}$ criterion between the measured dose by film and the planned dose. This means that the delivery of treatment was substantially verified.

There is no study on the comparison of QA of the treatment plans created using different collimator types. In addition, there is no guideline about QA of treatment delivery for Iris-collimator-based plans. In our study, we compared the QA results of plans generated using Iris and fixed collimator. Gamma-index pass rates using the $2 \% / 2 \mathrm{~mm}, 3 \% / 3 \mathrm{~mm}, 4 \% / 4 \mathrm{~mm}$, and $5 \% / 5 \mathrm{~mm}$ criteria for plans based on Iris collimator were found slightly lower than those for plans based on fixed collimator. Physical characteristics of Iris collimator that has two collimator banks with six leaves each may affect the results.

\section{Conclusion}

Pre-treatment validation for complex treatment plans, such as the CK treatment plan, has to be conducted. We performed the verification of the CK plans of ten patients with intracranial tumors using film dosimetry. This work indicates Gafchromic EBT2 film is a utilizable dosimeter for QA of the CK treatment plans. It is recommended to use the EBT2 film for verification of the $\mathrm{CK}$ treatment plans before delivery to the patient.

Peer-review: Externally peer-reviewed.

Conflict of Interest: None declared.

Financial Support: None declared.

Authorship contributions: Concept - C.K., G.K.; Design - C.K., G.K.; Supervision - C.K., H.B., G.K.; Materials C.K.; Data collection \&/or processing - C.K., U.A., N.D.K.; Analysis and/or interpretation - C.K., H.B., G.K.; Literature search - C.K., G.K.; Writing - C.K.; Critical review H.B., G.K.

\section{References}

1. Niranjan A, Bowden G, Flickinger JC, Lunsford LD. Gamma Knife Radiosurgery. In: Chin LS, Regine WF, editors. Principles and practice of stereotactic radiosurgery. 2nd ed. New York: Springer; 2015. p. 111-9.

2. Saw CB, Bao S, Li S. A review on the technical and dosimetric aspects of stereotactic body radiation therapy (SBRT). Journal of Radiation Oncology 2012;1(4):317-22.

3. Kilby W, Dooley JR, Kuduvalli G, Sayeh S, Maurer CR Jr. The CyberKnife Robotic Radiosurgery System in 2010. Technol Cancer Res Treat 2010;9(5):433-52.

4. Dieterich S, Cavedon C, Chuang CF, Cohen AB, Garrett JA, Lee CL, et al. Report of AAPM TG 135: quality assurance for robotic radiosurgery. Med Phys 2011;38(6):2914-36.

5. Reinhardt S, Würl M, Greubel C, Humble N, Wilkens JJ, Hillbrand M, et al. Investigation of EBT2 and EBT3 films for proton dosimetry in the 4-20 MeV energy range. Radiat Environ Biophys 2015;54(1):71-9.

6. Andrés C, del Castillo A, Tortosa R, Alonso D, Barquero R. A comprehensive study of the Gafchromic EBT2 radiochromic film. A comparison with EBT. Med Phys 2010;37(12):6271-8.

7. Kairn T, Hardcastle N, Kenny J, Meldrum R, Tomé WA, Aland T. EBT2 radiochromic film for quality assurance of complex IMRT treatments of the prostate: micro-collimated IMRT, RapidArc, and TomoTherapy. Australas Phys Eng Sci Med 2011;34(3):333-43. 
8. Cheung T, Butson MJ, Yu PK. Post-irradiation colouration of Gafchromic EBT radiochromic film. Phys Med Biol 2005;50(20):N281-5.

9. Low DA, Dempsey JF. Evaluation of the gamma dose distribution comparison method. Med Phys 2003;30(9):2455-64.

10. Chan MF, Zhang Q, Li J, Parhar P, Schupak K, Burman $C$. The verification of iPlan commissioning by radiochromic EBT2 films. Int J Med Phys Clin Eng Radiat Oncol 2012;1(1):1-7.

11. Budgell GJ, Perrin BA, Mott JH, Fairfoul J, Mackay RI. Quantitative analysis of patient-specific dosimetric IMRT verification. Phys Med Biol 2005;50(1):10319.
12. Agazaryan N, Solberg TD, DeMarco JJ. Patient specific quality assurance for the delivery of intensity modulated radiotherapy. J Appl Clin Med Phys 2003;4(1):40-50.

13. Mardirossian G, Koltermann P, Mirzasadeghi M, Muniruzzaman M.: Implementing patient specific plan QA for CyberKnife: comparison between two independent systems. Med Phys 2010;37:3257.

14. Bellec J, Delaby N, Jouyaux F, Perdrieux M, Bouvier J, Sorel S, et al. Plan delivery quality assurance for CyberKnife: Statistical process control analysis of 350 film-based patient-specific QAs. Phys Med 2017;39:50-8. 